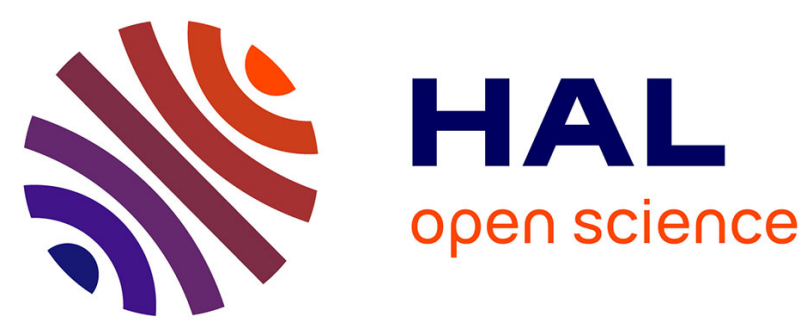

\title{
COVID-19 and mental health: A review and the role of telehealth and virtual reality
}

Ricardo Sainz-Fuertes, Joanmiquel Gelabert Mir, Josemaría Valderas, Kim

Bullock, Anne Giersch, Kai Vogeley, Ariadna Torres García, Marta Argiles

Huguet, Tamara Russell, Jack Hollingdale, et al.

\section{To cite this version:}

Ricardo Sainz-Fuertes, Joanmiquel Gelabert Mir, Josemaría Valderas, Kim Bullock, Anne Giersch, et al.. COVID-19 and mental health: A review and the role of telehealth and virtual reality. Digital Medicine, 2020,6 (2), pp.53. 10.4103/digm.digm_22_20. hal-03450332

\section{HAL Id: hal-03450332 \\ https://hal.science/hal-03450332}

Submitted on 25 Nov 2021

HAL is a multi-disciplinary open access archive for the deposit and dissemination of scientific research documents, whether they are published or not. The documents may come from teaching and research institutions in France or abroad, or from public or private research centers.
L'archive ouverte pluridisciplinaire HAL, est destinée au dépôt et à la diffusion de documents scientifiques de niveau recherche, publiés ou non, émanant des établissements d'enseignement et de recherche français ou étrangers, des laboratoires publics ou privés.

\section{(1)(1) $\$(0)$}

Distributed under a Creative Commons Attribution - NonCommercial - ShareAlikel 4.0 


\section{REVIEW ARTICLE}

\section{COVID-19 and mental health: A review and the role of telehealth and virtual reality}

Ricardo Sainz-Fuertes ${ }^{1 *}$, Joan Miquel Gelabert Mir ${ }^{1}$, Jose María Valderas ${ }^{2}$, Kim Bullock ${ }^{3}$, Anne Giersch ${ }^{4}$, Kai Vogeley ${ }^{5}$, Ariadna Torres García ${ }^{6}$, Marta Argiles Huguet ${ }^{7}$, Tamara Russell ${ }^{8}$, Jack Hollingdale ${ }^{9}$, Javier-David Lopez -Moriñigo ${ }^{10}$, Isidro Navarro Delgado ${ }^{11}$, Hugh Selsick ${ }^{12}$, Howard Gurr ${ }^{13}$, Tim Fitzpatrick ${ }^{14}$, Todd Maddox ${ }^{14}$

${ }^{1}$ Psious, Barcelona, Spain

${ }^{2}$ University of Exeter Medical School, Exeter, UK

${ }^{3}$ Department of Psychiatry and Behavioral Sciences, Stanford School of Medicine, USA

${ }^{4}$ University of Strasbourg, INSERM, University Hospital of Strasbourg, France

${ }^{5}$ Department of Psychiatry, University Hospital Cologne, Germany

${ }^{6}$ University Hospital Vall Hebron, Barcelona, Spain

${ }^{7}$ Psychosocial Intervention Team La Caixa, Barcelona, Spain

${ }^{8}$ Mindfulness Centre of Excellence, The Maudsley Hospital, London, UK

${ }^{9}$ The Michael Rutter Centre, The Maudsley Hospital, London, UK

${ }^{10}$ University Hospital "Fundación Jiménez Díaz", Madrid, Spain

${ }^{11}$ Polytechnic University of Catalonia, UPCBarcelonaTech, Barcelona, Spain

${ }^{12}$ Royal London Hospital for Integrated Medicine, London, UK

${ }^{13}$ The Doctors Gurr clinic, New York, USA

${ }^{14}$ IKONA Health, USA

\section{ABSTRACT}

On March 12, 2020, with more than 20,000 confirmed cases and almost 1000 deaths in the European Region, the World Health Organization classified the COVID-19 outbreak as a pandemic. As of August 15, 2020, there are 21.5 million confirmed cases of COVID-19 and over 766,000 deaths from the virus, worldwide. Most governments have imposed quarantine measures of varied degrees of strictness on their populations in attempts to stall the spread of the infection in their communities. However, the isolation may have inflicted long-term psychological injury to the general population and, in particular, to at-risk groups such as the elderly, the mentally ill, children, and frontline healthcare staff. In this article, we offer the most up-to-date review of the effects of COVID-19 confinement on all the disorders listed in the Diagnostic and Statistical Manual of Mental Disorders. We make data-driven predictions of the impact of COVID-19 confinement on mental health outcomes and discuss the potential role of telemedicine and virtual reality in mental health screening, diagnosis, treatment, and monitoring, thus improving the above outcomes in such a difficult time.

Keywords: COVID-19, Mental health, Telehealth, Virtual reality

\begin{tabular}{l}
\hline *Address for correspondence: \\
Dr. Ricardo Sainz-Fuertes, \\
Psious, Barcelona, Spain. \\
E-mail: drsainz@gmail.com \\
\hline \multicolumn{2}{c|}{ Access this article online } \\
\hline \multicolumn{2}{c|}{ Quick Response Code } & Website: \\
\hline
\end{tabular}

This is an open access journal, and articles are distributed under the terms of the Creative Commons Attribution-NonCommercial-ShareAlike 4.0 License, which allows others to remix, tweak, and build upon the work non-commercially, as long as appropriate credit is given and the new creations are licensed under the identical terms.

For reprints contact: WKHLRPMedknow_reprints@ @olterskluwer.com

How to cite this article: Sainz-Fuertes R, Gelabert JM, Valderas JM, Bullock K, Giersch A, Vogeley K, et al. COVID-19 and mental health: A review and the role of telehealth and virtual reality. Digit Med 2020;6:53-66.

Submitted: 01-Sep-2020 Accepted: $26-\mathrm{Nov}-2020$
Revised: 21-Sep-2020 Published: $19-$ Nov-2021 
Sainz-Fuertes, et al.: COVID-19 and mental health

\section{INTRODUCTION: THE COVID-19 PANDEMIC}

The official name for the disease caused by the 2019 novel coronavirus outbreak, which was first identified in Wuhan (China), is COVID-19. Specifically, COVID-19 is caused by the severe acute respiratory syndrome-coronavirus-2 (SARS-CoV-2), a beta-coronavirus, like Middle East respiratory syndrome-CoV and SARS-CoV, which had also caused a respiratory syndrome previously. The onset and duration of viral shedding and the period of infectiousness for COVID-19 remain to be established although its incubation period has been estimated to range from 2 to 14 days. ${ }^{[1]}$

The highest risk groups are elderly people and those who live in a nursing home or long-term care facility. COVID-19 also impacts all age groups with underlying medical conditions such as chronic lung disease or asthma, serious heart condition, compromised immune systems, severe obesity, diabetes, and renal failure undergoing dialysis and those with liver diseases. Further, of concern, frontline staff including healthcare workers, emergency services personnel, and nursing homes staff have been highly affected. ${ }^{[1]}$

As of September 1, 2020, there is no specific or effective, evidence-based treatment or vaccine for COVID-19; hence, acquired immunity cannot be assured. Moreover, there have been worrying reports of potential long-term neurodegenerative sequelae and neuro-immune dysregulation after recovery from COVID-19, and children appear to present with signs of autoimmune inflammatory reaction to the virus. ${ }^{[1]}$ According to the World Health Organization (WHO), the COVID-19 pandemic outbreak size and the subsequent negative impact on our society, including a significant economic disruption, have made this disease more catastrophic than any other that our generation has had to live through. ${ }^{[2]}$ Almost every country has been affected, and even the most resilient healthcare systems have struggled treating a large number of patients. It is estimated that around $20 \%$ of cases are severe or critical, with a crude clinical case-fatality rate of over 3\%. This rate is higher in older age groups and in those with certain underlying medical conditions, which has also disproportionately affected healthcare workers and people living in residential care settings. Moreover, the widespread extent of home confinement and other contention/lockdown measures implemented in an attempt to stem the rate of infection have had broad and deep socioeconomic consequences. ${ }^{[2]}$ The
WHO has suggested that a global coordinated strategic response is needed. ${ }^{[3]}$

\section{LESSONS FROM THE PAST}

Effective quarantine measures, including confinement, cannot be implemented without a cost, in terms of both financial and health outcomes. A 2004 Canadian study ${ }^{[4]}$ examined the psychological effects of quarantine due to SARS control measures on Toronto residents through a cross-sectional, web-based survey, which was completed by 129 persons who spend a median duration of 10 days in quarantine. A high prevalence of psychological distress was identified with $28.9 \%$ having symptoms of posttraumatic stress disorder (PTSD) and 31.2\% having symptoms of depression. Longer durations of quarantine, acquaintance with or direct exposure to someone with a diagnosis of SARS were also associated with an increased prevalence of PTSD symptoms, which tended to worsen as the household income fell. ${ }^{[4]}$

Sprang and Silman ${ }^{[5]}$ measured self-reported stress in confined families using the University of California at Los Angeles Posttraumatic Stress Disorder Reaction Index Parent Version and the PTSD Check List Civilian Version and found that criteria for PTSD were met by $25 \%$ of parents and $30 \%$ of children (based on parental reports). ${ }^{[5]}$

Beaglehole et al. ${ }^{[6]}$ conducted a systematic review and meta-analysis to explore the effects of natural disasters on mental health. They identified 41 studies, 27 of which were meta-analyzed and found psychological distress and psychiatric disorders increased after natural disasters, especially rates of PTSD and depression, although changes in anxiety and alcohol misuse/dependence rates were not significant.

Brooks et al., ${ }^{[]]}$a review published in the Lancet, found that quarantine measures result in psychological distress, although this can be attenuated by some measures. They found consistent evidence of trauma following the quarantine measures in hospital staff immediately after the quarantine period ( 9 days), who were more likely to report a wide range of psychological distress symptoms, such as exhaustion, detachment from others, anxiety when dealing with febrile patients, irritability, insomnia, poor concentration and indecisiveness, deteriorating work performance, and reluctance to work or consideration of resignation. Being quarantined was also a predictor of long-term PTSD in hospital employees (at 3 years). Quarantined children were also found to be at risk of trauma, showing posttraumatic stress scores four times higher than those who had not 
Sainz-Fuertes, et al.: COVID-19 and mental health

been quarantined, and $28 \%$ of the parents quarantined reported sufficient symptoms to warrant a diagnosis of a trauma-related mental health disorder, compared with $6 \%$ of parents who were not quarantined. ${ }^{[7]}$

Interestingly, this review included a study examining symptoms of depression 3 years after quarantine involving hospital staff and found $9 \%$ of the sample to report severe depressive symptoms. In this group, nearly $60 \%$ had been quarantined, whereas only $15 \%$ of the mild depressive symptoms group had been quarantined. [7] People quarantined also reported fear $(>20 \%)$, nervousness $(18 \%)$, sadness $(18 \%)$, and guilt $(10 \%)$, as well as a range of other psychological responses to quarantine, such as confusion, anger, grief, numbness, and insomnia. More worryingly, in healthcare workers, alcohol abuse or dependency symptoms were positively associated with having been quarantined 3 years after the end of the quarantine period. Quarantine was also significantly and positively associated with avoidance behaviors, such as minimizing direct contact with patients and not reporting to work. ${ }^{[7]}$

These findings were replicated by a literature review on the effects of quarantine and isolation for infection prevention on mental health outcomes that revealed high levels of depression, anxiety, mood disorders, psychological distress, PTSD, insomnia, fear, stigmatization, low selfesteem, lack of self-control, and other adverse mental health outcomes among patients, informal caregivers, and healthcare providers who experienced quarantine or isolation. The authors suggested multipronged interventions, including policy measures for strengthening mental health services globally and promoting psychosocial well-being among high-risk populations, to mitigate the effects of containment measures on healthcare providers' mental health, thus lessening suboptimal performance in workplaces. ${ }^{[8]}$

Given the impact of quarantine and isolation on mental health outcomes in the general population, concerns have been voiced regarding vulnerable groups. A recent position paper published in the Lancet is especially relevant. ${ }^{[9]}$ The authors suggest a number of potential measures to support the most vulnerable groups under pandemic conditions, frontline healthcare and social care staff, those with pre-existing mental health issues, young people (aged $\leq 18$ years), and older adults (aged $\geq 65$ years). These measures include, but are not limited to:

- Deploying interventions that can be delivered under pandemic conditions to reduce mental health issues and boost well-being
- Developing methods for promoting more successful adherence to behavioral advice about COVID-19 while enabling mental well-being and minimizing distress. ${ }^{[0]}$

\section{THE COVID-19 PANDEMIC EARLY LEARNING AND MENTAL HEALTH EFFECTS}

Evidence of the impact of COVID-19 on mental health emerged initially in China, which was later replicated in other countries. Based on a sample of 7236 Chinese participants, Huang and Zhao ${ }^{[10]}$ designed a web-based, cross-sectional survey to collect the data from volunteers using validated instruments such as the Generalized Anxiety Disorder-7 (GAD-7), Center for Epidemiology Scale for Depression, and Pittsburgh Sleep Quality Index. Overall, the prevalence of GAD (35.1\%), depressive symptoms (20.1\%), and poor sleep quality $(18.2 \%)$ was high. Specifically, two main findings emerged from the study. First, adults aged 35 years and younger reported having a higher prevalence of GAD and depressive symptoms than older people $(P<0.001)$. Second, compared with other occupational groups, healthcare workers showed the highest rate of poor sleep quality $(P<0.001) \cdot{ }^{[10]}$

Rosen et al..$^{[1]}$ conducted a survey in the US among approximately 1250 quarantined New York city residents. Rosen $e t$ al. measured distress using the Subjective Units of Distress Scale and anxiety using the Beck Anxiety Inventory. The sample was comprised of 303 individuals and the mean levels of distress were shown to be heightened and sustained, with $69 \%$ of participants reporting moderate-to-severe distress and 25\% indicating level of anxiety/distress that significantly impaired functioning. The authors suggested that access to professional mental healthcare as well as behavioral interventions should be prioritized. ${ }^{[11]}$

In one multicenter survey involving $n=1563$ medical staff in Guangzhou, China, Liu et al. found high prevalence of depression (50.7\%), anxiety $(44.7 \%)$, insomnia (36.1\%), and stress-related symptoms (73.4\%). ${ }^{[12]}$ The authors concluded that access to professional mental health care as well as behavioral interventions should be prioritized. ${ }^{[11]}$

Consistent with this, Galea et al. ${ }^{[13]}$ warned that there would be substantial increases in mood disorders, substance use, loneliness, and domestic violence and high levels of child abuse in the context of the COVID-19 pandemic and they suggested that a plan was needed to 
Sainz-Fuertes, et al.: COVID-19 and mental health

address the inevitable mental health crisis using digital technologies.

In a recent survey, ${ }^{[14]} 3550$ Spanish adults' symptoms of anxiety, depression, and stress were analyzed using the 21-item version of the Depression Anxiety Stress Scale, and symptoms of PTSD were assessed with the Impact of Event Scale. A large percentage of respondents reported high levels of anxiety (32.4\%), depression (44.1\%), and stress $(37.0 \%)$. Furthermore, symptomatic scores indicating psychological stress were found in $88.6 \%$ of respondents.

A systematic review and metaanalysis conducted by Rogers et al. ${ }^{[15]}$ advised clinicians to be mindful of the possibility of depression, anxiety, fatigue, post-traumatic stress disorder, and rarer neuropsychiatric syndromes in the longer term in a proportion of patients infected by SARS-CoV-2.

Taken together, these findings suggest significant increased risk for mental disorders in the general population and for those already suffering from mental health issues that are directly linked to the COVID-19 pandemic. Given the need for isolation and physical distancing due to the pandemic and the fact that this exacerbates mental health issues, it is clear that digital technologies should be considered to address this need.

\section{THE IMPACT OF NATURAL DISASTERS AND CONFINEMENT ON PEOPLE WITH MENTAL ILLNESSES}

As the review above suggests, quarantine strongly affects people with mental illness, healthcare workers in hospitals and laboratories, and volunteers and social service personnel. ${ }^{[16]}$ Psychiatrists should therefore educate people and encourage them to engage in healthpromoting behaviors, assist in the integration of mental and healthcare, empower patients and families, and regularly monitor mental health of healthcare providers, including delivering care as appropriate. ${ }^{[16]}$

Given the upcoming significant financial constraints as a result of the COVID-19 pandemic, it is envisaged that these interventions will have to be delivered remotely, using telehealth and other solutions such as virtual reality (VR). Therefore, guidelines on how to conduct online telepsychiatry consultations are needed. These have been published by The Royal College of Psychiatrists in the $\mathrm{UK}^{[17]}$ and by the American Psychiatry Association, in the US. ${ }^{[18]}$ The $\mathrm{WHO}^{[19]}$ and
Centers for Disease Control and Prevention $(C D C)^{[20]}$ have issued the guidance and resources to support the population and ensure resilience in societies during this pandemic.

Before turning to a discussion of the role of telehealth and VR in addressing mental health concerns due to the COVID-19 pandemic, we generate a comprehensive set of predictions regarding the impact of COVID-19 on all Diagnostic and Statistical Manual of Mental Disorders (DSM) mental disorders and offer solutions for sustainability and treatment.

\section{NEURODEVELOPMENTAL DISORDERS}

Neurodevelopmental conditions include autism spectrum disorder, attention deficit hyperactivity disorder, and intellectual disability. These life-long conditions are associated with varying degrees of cognitive, emotional, behavioral, social, and functional impairment and share a number of diagnostic symptoms. As such, the effective treatment and management of such conditions may involve a number of different agencies and systems of support including familial, educational, mental healthcare, and social care services.

However, the COVID-19 pandemic has resulted in worldwide government-enforced lockdowns. This has caused considerable disruption to the systems of support and typical access to appropriate services for young people and adults with neurodevelopmental conditions. Despite services adapting their provisions to accommodate the changes brought about by COVID-19, such as using telephone or video technology, the face-to-face support, including therapy, education, medication reviews, and respite that are relied upon by many, has been dramatically reduced.

In response to these limitations, a number of management strategies have been identified by various groups across Europe for young people and their families to consider during this challenging period [detailed alleviation strategies can be found in Table 1].

Young people and adults diagnosed with neurodevelopmental disorders are at an increased risk of experiencing low mood and anxiety when routines are disrupted. Reducing uncertainty, such as introducing schedules and structure during this challenging time, can support their mood and unhelpful behavior. It is essential that all relevant services continue to engage with young people and families via the telephone and online 
Sainz-Fuertes, et al.: COVID-19 and mental health

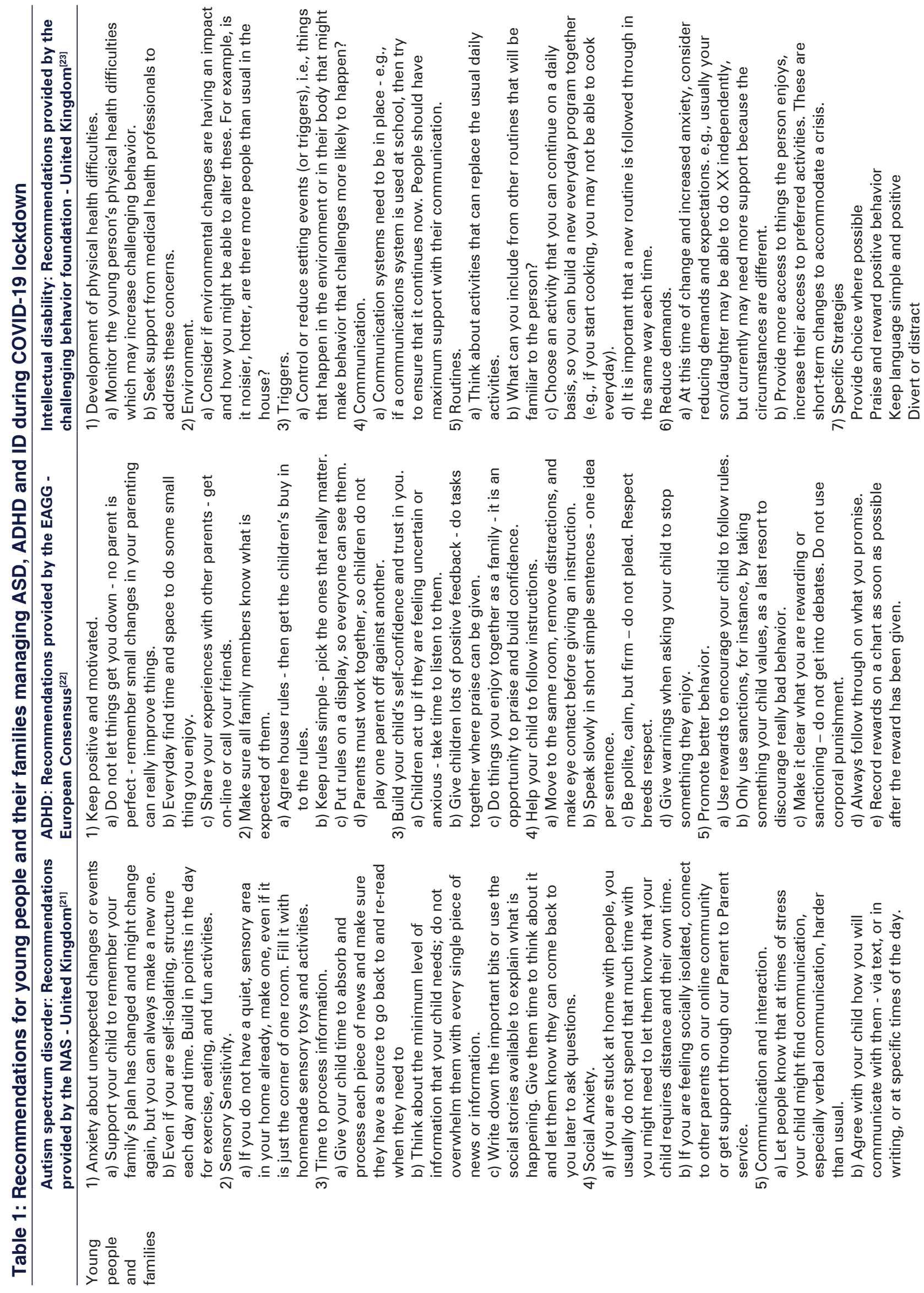


Sainz-Fuertes, et al.: COVID-19 and mental health

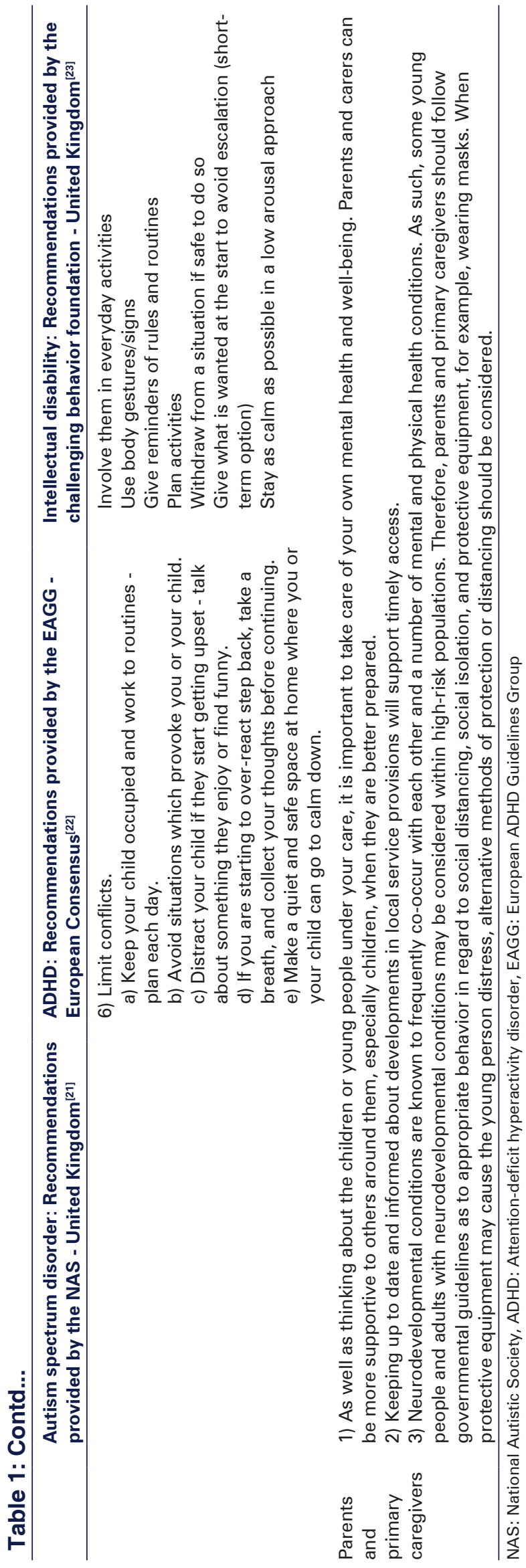

technologies to ensure continued support. ${ }^{[2]}$ In regard to ensuring continued access and compliance to medication, effective communication channels between caregivers, pharmacists, and prescribers and implementing solutions for remote monitoring should be developed. ${ }^{[22]}$

Furthermore, for parents of young people and adults with neurodevelopmental conditions, timely and accessible information is essential to ensure that they are up to date with changes in the lockdown restrictions. This may include information and guidance around changes in personal protective equipment, restrictions around visiting others or receiving visitors, both social and professional, changing roles of professionals or professional services, such as social care or clinical support, including mental health support and medication prescriptions. Inconsistency in information or obstacles accessing relevant information may further increase uncertainty and anxiety for families and adults with neurodevelopmental conditions. Systems involved in their care such as neurodevelopmental or mental health services, general practitioners, social care, educational providers, or employers should ensure that they are informed with the most up-to-date information. In the absence of typical face-to-face appointments, technological strategies should be employed where appropriate.

\section{SCHIZOPHRENIA SPECTRUM AND OTHER PSYCHOTIC DISORDERS}

Patients suffering from psychotic disorders are at higher risk of infectious diseases and tend to have worse medical outcomes than the general population. In particular, most of these patients are affected by clinical comorbidities in the cardiovascular system and metabolic disorders. ${ }^{[25]}$ These individuals often have a heightened sense of fear, stress, and boredom associated with the lack of stimulation brought by compulsory isolation, poor compliance, and loss to followup by their healthcare services. In addition, lack of judgment and poor self-care (self-neglect) seem to hinder compliance with health recommendations which put patients, families, and health professionals at higher risk. ${ }^{[2]}$

The authors suggest a series of recommendations for patients, health professionals, and families of people with schizophrenia.

1. Schizophrenia patients should follow the same health instructions and receive the same treatment as clinical high-risk groups for COVID-19, i.e., equality and justice.

2. General practitioners' screening COVID-19 patients should pay special attention to those with schizophrenia who may struggle to report respiratory symptoms. 
Sainz-Fuertes, et al.: COVID-19 and mental health

3. Mental health professionals should remotely monitor unstable outpatients, for which telehealth (internet-based) technologies may be useful.

4. Mental health services should inform patients and families of all procedures to be followed during this period and should provide clear remote contact channels to minimize overcrowding and exposure to potential sources of infection in hospital settings. However, families should also monitor for signs of relapse and contact mental health services as soon as possible.

5. Professionals and families should promote and monitor adherence to antipsychotic medication, ensuring access to prescriptions, and organizing a delivery system, including clozapine.

6. To reduce emotional distress - and possibly psychotic relapse - families and patients should be advised to:

a. Anticipate stress reactions and be informed about them by professionals.

b. Prepare for quarantine, which can be better adhered to if patients and families receive more information about COVID-19, about the benefits of quarantine (both personally and in terms of public health), and about quarantine procedures (such as adequate supplies of food and medication).

c. Limit media exposure, since overexposure to broadcasts of stressful situations has been linked to negative mental health outcomes, and avoid misinformation.

In a rapid review of the evidence of contemporary epidemic and pandemic research to evaluate the potential impact of COVID-19 on psychosis, Brown et al. indicated that there is moderate (albeit of low quality) evidence suggesting an increased risk of coronavirus related to psychosis in people exposed to a virus during the pandemics as previous research showed that between $0.9 \%$ and $4.0 \%$ of people exposed developed psychosis compared with a median incidence rate of 15.2 in 100,000. This is likely to be associated with steroids or viral exposure, pre-existing vulnerability, and/or psychosocial stress and should be treated with low doses of antipsychotic medication, particularly aripiprazole, which seems to have been effective in patients with emerging infectious disease. ${ }^{[2]}$

No evidence of changes in the form and content of psychotic symptoms, impact on the physical health of people with psychosis, rates of suicide or attempted suicide, incidence of homelessness, unemployment, and domestic violence was found. Recommendations to policymakers and service managers were to consider providing some forms of community support using telehealth technology to protect their staff. ${ }^{[26]}$

\section{BIPOLAR AND RELATED DISORDERS}

Patients with bipolar disorders are likely to suffer from the disruption of medical and health services, increased strain, disrupted sleep and routines, and stigma. ${ }^{[2]}$ These patients are also more likely to suffer from infections, metabolic disorders, and medical comorbidities than the general population. Medical treatment for COVID-19 is multifaceted (containment and hygiene measures, confinement, physical distancing, and use of antibiotics). As a result, some patients with bipolar disorder may find it difficult to follow and to adhere to while maintaining their specific mental health pharmacological and behavioral treatment. Treatment plans will require active monitoring to prevent adverse side effects and/or lack of response. ${ }^{[28]}$ Finally, these subjects are likely to face a double stigma stemming from their mental and health problems. ${ }^{[27]}$

We need to reconsider novel ways to facilitate access to therapeutic interventions for this patient population. Faceto-face visits could be replaced by telehealth solutions that facilitate assessment, monitoring, diagnosis, and treatment, including issuing prescriptions. In addition, psychoeducation programs, mindfulness sessions, sleep hygiene recommendations, and online support resources specific for bipolar patients could help ameliorate the effects of the pandemic and its forced confinement measures. ${ }^{[29]}$

Organizations such as the International Bipolar Foundation $^{[30]}$ and Bipolar $\mathrm{UK}^{[31]}$ have published practical recommendations on their websites that include:

1. Having adequate supply of medication.

2. Keeping track of manic and depressive episodes.

3. Looking after physical health: Eat well, stay hydrated, get enough sleep, and exercise.

4. Being mindful of lithium toxicity and virus infection.

\section{DEPRESSIVE AND ANXIETY DISORDERS}

Social containment measures implemented to control the COVID-19 pandemic are linked with an increased risk of depression and anxiety disorders, ${ }^{[6,7]}$ and those with established mood disorders are more likely to relapse as a result. Of major concern for these patients are the social containment measures implemented in an attempt to control the pandemic. These patients have demonstrated $21 \%$ higher pooled population attributable risk of suicide than their counterparts from the general population. ${ }^{[32]}$ 
Sainz-Fuertes, et al.: COVID-19 and mental health

According to the WHO, suicide accounts for approximately 800,000 deaths/year. The COVID-19 pandemic has resulted in unprecedented increases in unemployment and social isolation, both of which are well-established risk factors for suicide, nationally and globally.

In a recent paper, Weems $e t$ al. estimated the increased rate of suicide worldwide to be close to 50,000 individuals as a result of increased unemployment and social isolation brought by containment measures and recommended to weigh the efforts to stop the spread of COVID-19 against these potential collateral mental health effects. ${ }^{[33]}$ This increase in suicide risk is likely to affect the elderly particularly as pointed out by Yip et al. in previous research conducted in SARS-related older adult suicide victims. ${ }^{[34]}$ The suicide motives were mainly associated with stress over fears of being a burden to their families, social disengagement, mental stress and anxiety related to fear of getting sick and dying, fear of losing people, fear of unpredictable shortages, and economic disaster at the time of the SARS epidemic. Hence, tackling stigma, which requires an integrative approach involving different elements of society, may significantly reduce suicide risk associated with the COVID-19 pandemic.

\section{OBSESSIVE-COMPULSIVE AND RELATED DISORDERS}

Patients with obsessive-compulsive disorder (OCD) symptoms related to cleanliness and fear of infection are among the top affected patient population in times of pandemics. COVID-19-related anxiety is likely to exacerbate existing obsessive fears of contamination, which may lead to potentially harmful compulsive acts ${ }^{[35]}$ or justification of their symptoms, thereby challenging their therapeutic program.

A working group of clinical experts from the International College of Obsessive Compulsive Spectrum Disorders and the Obsessive-Compulsive Research Network of the European College of Neuropsychopharmacology has produced a pragmatic guidance for clinicians to help them manage this patient population. ${ }^{[35]}$ A summary is presented below with strong encouragement to review the full guidance on the original paper:

1. Take a compassionate calming approach. Use telemedicine, including telephone or video calls.

2. Careful history-taking. Confirm the diagnosis of OCD, paying particular attention to other related disorders such as hypochondriasis, which are likely to be most affected by COVID-19.
3. Suicide risk assessment. Although OCD has not been associated with a high risk for suicide, the presence of with severe obsessions, comorbid depression, bipolar disorder, impulse control disorders, substance use disorders, personality, and eating disorders should be properly evaluated, which may increase the risk.

4. Provide psychoeducation with balanced information about known risk factors and impact of COVID-19 on physical and mental health.

5. Enquire about internet usage and news consumption. Suggest trusted sources to avoid myths, rumors, and misinformation:

a. Health education websites:

i. WHO (https://www.who.int/health-topics/ coronavirus\#tab=tab_1)

ii. CDC (https://www.cdc.gov/ coronavirus/2019-ncov/infection-control/ hcp-hand-sanitizer.html)

iii. Center for Health Security, Johns Hopkins University (http://www. centerforhealthsecurity.org/resourcees/ COVID-19/index.html).

b. Handwashing videos:

i. National Health Service (https://www.nhs. uk/video/pages/how-to-wash-hands.aspx)

ii. CDC videos (https://www.youtube.com/ watch?v=3EoAyQu3LIs).

1. If OCD symptoms are the main problems:

a. Review medication status as a priority: Pharmacotherapy should be the first option for adults and children with OCD with contamination, washing or cleaning symptoms during the COVID-19 pandemic.

b. Review and risk: Assess the cognitive-behavioral therapy (CBT) plan, including considerations around feasibility and compliance with the government safety guidance. We suggest therapists to regularly check, e.g., by phone or digitally, those OCD patients more likely to engage in particularly harmful decontamination rituals or behaviors. Video calls should be recommended, where possible.

c. Deep brain stimulation (DBS): Delaying the implantation of electrodes in OCD patients waiting for DBS until the outbreak is over, although it is worth noting that these subjects suffer from highly disabling treatment-resistant OCD symptoms.

d. Social and occupational care: Patients under quarantine or staying at home under national restrictions are at great risk of circadian rhythm disruption which could increase anxiety and worsen OCD symptoms. Offer guidance 
Sainz-Fuertes, et al.: COVID-19 and mental health

regarding a rational amount of time spent listening to news may help the isolated patient to overcome loneliness. Regular communication with friends, family members, and loved ones regardless of distance, for instance, via online platforms, such as Facetime, Skype, and Zoom, is essential. Patients and family members should also be encouraged to keep weight under control.

e. Carer support: Family members and cares are at increased risk of stress-related disorders and may therefore need additional support.

\section{TRAUMA- AND STRESSOR-RELATED DISORDERS AND DISSOCIATIVE DISORDERS}

The profound loss, stress, and fear associated with COVID-19 are likely to exacerbate previous mental health disorders and contribute to the onset of new stress-related disorders. ${ }^{[36]}$ This pandemic is exerting unusual pressure on our collective healthcare, political, economic, and social-welfare systems given the profound loss, stress, and fear associated with COVID-19 will likely exacerbate existing mental health disorders and contribute to the onset of new stress-related disorders for many. ${ }^{[36]}$ Patients suffering from trauma and/or those developing de novo symptoms might benefit from concerted efforts targeting diagnostic process, prevention, public outreach, and communication by liaising with mental and physical healthcare services and designing "COVID-19 and Trauma" research projects. ${ }^{[36]}$

\section{SOMATIC SYMPTOM AND RELATED DISORDERS}

Little is known about the effects of COVID-19 on patients suffering from somatic symptoms/disorders. A Chinese large-scale survey of psychological distress in the general population during the COVID-19 pandemic showed that almost $35 \%$ of respondents had experience psychological distress (described as experiencing either anxiety, depression, specific phobias, cognitive change, avoidance and compulsive behavior, physical symptoms, and loss of social functioning) during 7 days before the survey being conducted. ${ }^{[37]}$ Another study revealed that patients who experienced COVID-19 were more likely to have depressed mood and somatic symptoms in the subitems of the PHQ9, compared to individuals under quarantine. ${ }^{[38]}$

\section{FEEDING AND EATING DISORDERS}

In brief, eating disorders are characterized by food restriction. Food insecurity and panic buying both have occurred during the COVID-19 pandemic and may have a detrimental effect on patients with eating disorders. ${ }^{[39]}$ In keeping with this, Touyz et al., writing in the Journal of Eating disorders, warned us that these patients have a complex and difficult relationship with food and that food insecurity and panic buying will worsen it. Exceptionally, low-weight people with anorexia nervosa may be particularly vulnerable to COVID-19 because of their compromised physical health. People with bulimia nervosa and binge eating disorder, however, might encounter increased family conflict, higher risk of depression and anxiety, selfharm behaviors, or even suicidality when their bingeing behavior impacts on the family's food at a time when restocking is problematic. ${ }^{[39]}$ Healthcare systems should address the special needs of eating disorder patients. In the short term, standard treatment approaches should be re-considered and CBT group programs through video conferencing delivered, which have proven effective under similar circumstances. ${ }^{[39,40]}$

\section{SLEEP-WAKE DISORDERS}

The prevalence of insomnia diagnoses according to the DSM-IV Classification is at $6 \% .{ }^{[4]}$ Mental disorders and organic diseases can increase the risk of insomnia. On the other hand, individuals with insomnia are more likely to have a major depressive illness and longitudinal studies have shown that the persistence of insomnia is associated with the appearance of a new depressive episode. ${ }^{[41]} \mathrm{We}$ now know that over a third of the frontline medical staff who first attended patients in China suffered insomnia symptoms during the COVID-19 outbreak ${ }^{[42]}$ and that the disruption brought by changes in social mobility, exercise, and work patterns resulted in increased risk of anxiety-, depression-, and trauma-related symptomatology $;^{[10]}$ and hence, it is likely that those patients with existing sleepwake disorders will see their symptoms worsened by the physical and mental health impact of the pandemic. ${ }^{[43]}$

\section{SUBSTANCE-RELATED AND ADDICTIVE DISORDERS}

COVID-19 could be an especially serious threat to those who smoke tobacco or marijuana or who vape. People with opioid use disorder and methamphetamine use disorder are also high-risk COVID-19 groups due to the respiratory effects of these drugs. ${ }^{[4]}$ In addition, people with substance use disorders tend to have limited access to healthcare services and high levels of housing and employment insecurity and incarceration, all of which are well-known risk factors of suicide. As alluded to above, access to mental healthcare and substance use disorders 
Sainz-Fuertes, et al.: COVID-19 and mental health

treatment is likely to be hindered by the COVID-19 pandemic. The use of telemedicine might mitigate this, however, which is particularly relevant in such a vulnerable population. ${ }^{[45]}$

\section{NEUROCOGNITIVE DISORDERS}

Individuals with Alzheimer's disease and related dementia (ADRD) are a very high-risk group for COVID-19 infection in terms of morbidity and mortality because of their age, frequent medical comorbidities and difficulties in understanding and complying with public health recommendations due to cognitive impairment or comorbid mood or psychotic symptoms. ${ }^{[46]}$ Of concern, nonurgent clinical management of these patients may be postponed, while healthcare systems struggle to cope with the unusual demand in emergency services. Communities will find it harder to support individuals with ADRD, both informally through relatives and formally through professional caregivers, because of confinement and containment measures. In addition, financial adversity will hit harder those with limited resources and families should be supported and provided with essential services. Mitigation strategies, such as telehealth solutions, might be necessary to support ADRD patients, carers, and clinicians. ${ }^{[46]}$ Support for people living with dementia and caregivers should be delivered collaboratively by multidisciplinary health and social care teams which meet patient physical, mental health, and psychosocial needs. Self-help interventions such as relaxation and meditation exercises should be encouraged. Behavioral management and support could be delivered through telephone and psychological interventions could be provided online as these emerging technologies can be harnessed to secure social support networks and a sense of belonging. ${ }^{[4]}$ In summary, the impact of COVID-19 on these patients will depend on a variety of factors, such as the severity of cognitive impairment, whether there is associated agitation, type of residential placement, existence of informal and formal care support networks, availability of resources, and how strict public health measures deployed to control the pandemic are. Therefore, making simple generic recommendations appears to be impractical. ${ }^{[46]}$

\section{SUMMARY}

We are living through the worst global natural disaster of our generation. The likely adverse effects of the pandemic on population mental health will be exacerbated by fear, self-isolation, and the physical distancing imposed by public health organizations until a vaccine or specific treatment is found. In other words, COVID-19 core prevention measures, namely physical distancing and confinement, may succeed at the price of worsening mental health outcomes. These mental health problems will be experienced by the general population, and more acutely so, by those with high levels of exposure to COVID-19 such as frontline healthcare workers, those who eventually develop severe symptoms of the infection by SARS-COV-2, and those with previous mental health problems.

The COVID-19 pandemic will cause untold psychological distress and lead many people to develop mental health problems which are likely to become chronic in some cases and might even peak much later than the actual pandemic, which is known as the post-COVID fourth wave. Those with premorbid mental disorders might experience worsening of symptoms, while others might develop new-onset mental health problems, especially depression, anxiety, and posttraumatic stress. ${ }^{[48]}$

Strategies should be therefore prioritized, including a concerted effort between public and private institutions providing evidence-based online resources and free of cost scalable interventions.

These therapeutic interventions should target specific atrisk populations such as healthcare staff, particularly those working in intensive care units, those with physical and mental comorbidities and chronic conditions, children and parents, and those with low levels of education or who suffer from bereavement, perceive themselves at risk, or live in outbreak hotspots. ${ }^{[4]}$ Stepped care (i.e., delivering the most effective, yet least resource-heavy treatment to patients in need and then stepping up to more resource-heavy treatment based on patients' needs) may thus be a useful approach to meeting mental health needs during the COVID-19 pandemic. ${ }^{[13]}$

\section{THE ROLE OF TELEHEALTH AND VIRTUAL REALITY IN REDUCING COVID-19 MENTAL HEALTH BURDEN}

As the comprehensive review above suggests, the COVID-19 pandemic is having a huge negative impact on the general population, especially frontline workers and those with mental health issues. Isolation, fear, and uncertainty are leading to mental health issues in the general public and are exacerbating problems for those suffering from mental health issues. Digital therapeutic technologies offer a cost-effective, scalable solution to address these mental health problems as part of a multipronged approach. 
Sainz-Fuertes, et al.: COVID-19 and mental health

For example, video-based telemedicine in which a patient uses a flat-screen video service to "meet" with their mental health professional can serve as a stopgap and in some cases a replacement for in person visits. Although physical contact is precluded, with video, the mental health professional can still read verbal and nonverbal cues and can undertake a synchronous interaction. If wearable sensors are incorporated, physiological measures can be shared with the mental health professional in real time.

There is a growing body of evidence supporting the effectiveness of video-based mental health solutions, especially in the context of depression, anxiety, and PTSD. This digital technology can be used for diagnostic screening, treatment, and monitoring as well as to deliver psychoeducation, support, and relevant trustworthy information. ${ }^{[50]}$

\section{VIRTUAL REALITY FOR BEHAVIORAL AND MENTAL HEALTH}

VR-based behavioral and mental health solutions offer an innovative complement to video-based solutions or in person mental health solutions. VR, unlike any other digital therapeutic technology, broadly engages multiple systems in the brain, including cognitive, emotional, behavioral, and experiential learning systems. ${ }^{[51]}$ VR leads to a sense of immersion and presence that can only be matched by real-world experience. Thus, VR can transport a patient into any virtual, realistic environment to address multiple mental health issues with consistency, scalability, and $24 / 7$ availability. No other digital therapeutic technology has this power and potential.

Innovative approaches to the use of VR for several treatments have been developed. These include approaches to reduce stress and promote relaxation, ${ }^{[5]}$ to treat PTSD ${ }^{[53]}$ and anxiety disorders, ${ }^{[54]}$ to facilitate pain management, ${ }^{[5]}$ treat addiction ${ }^{[56]}$ or psychosis, ${ }^{[57]}$ and as assessment tools. ${ }^{[58]}$ There are hundreds of other solutions currently in the development.

VR has been used extensively to complement in person therapy for anxiety, including public speaking, phobias, fear of flying, and panic attacks, to name a few. Patients are immersed in VR while physically present with the mental health professional. The mental health professional can track physiological and subjective metrics and can increase or decrease the anxiety-provoking exposure. With the recent COVID-19 pandemic, mental health professionals and VR mental health solution providers are exploring remote applications of these same anxiety reducing solutions. Patients can be provided with VR headsets (or the inexpensive Google Cardboard) and can be immersed in mental health relevant environments from the comfort of their own home while connected via some telemedicine platform with their therapist. Patients can be assigned "homework" as well, to speed progress on the therapeutic journey.

Another fascinating application of VR is in social and interpersonal skills and eating disorders. The ability to "walk a mile in someone else's shoes" in VR is especially important for interpersonal and social skills development to allow the patient to "experience" how they are perceived by others. Analogously, the ability to "walk a mile in your own shoes" in VR can provide insights to a patient suffering from an eating disorder. Patients can gain direct experience with their distorted of body image and can help them correct these distortions in the interest of developing a more realistic perception.

Across all of these applications, one primary strength of VR is the consistency of experience, the scalability of the application, and the $24 / 7$ availability. All of these behavioral and mental health issues are issues in "learning." It is well established from over a century of basic science research on learning ${ }^{[59]}$ that learning and the relevant behavior change require hundreds if not thousands of repetitions. VR provides the opportunity to meet this need.

\section{EMBODIED VIRTUAL REALITY}

The future of VR in mental health (and many other applications) is in the full embodiment in VR. The majority of VR applications use visual and auditory cues to create the sense of immersion and presence. This is highly effective in behavioral and mental health; however, as haptic, tactile, and olfactory senses are incorporated, the feeling of embodiment will increase. The potential here is huge. Embodied VR has the potential to facilitate remote therapies that can be more consistent, scalable, and available. Embodied VR can more directly address body image disorders and facilitate the development of appropriate eating behaviors. Embodied VR can directly address the foundational problems associated with isolation and loneliness brought on by the pandemic. Embodied VR is still relatively novel, and more empirical and technical work is in order. Even so, the value is clear and the evidence is growing. 
Sainz-Fuertes, et al.: COVID-19 and mental health

The need for these digital therapeutic technologies and their growing value is not going unnoticed by the medical community. The Journal of the American Medical Association recently published an opinion piece arguing for the creation of a new medical specialty focused on virtual care called "medical virtualist." [60] Nowadays, the information and communication technology (ICT) has evolved to provide enough resources (software and hardware) to make it possible. The success to technology-based services is not determined by ICT access alone but also by ease of use, perceived value, and workflow optimization. ${ }^{[59]}$ The coordination of virtual care with home visits, remote monitoring, and simultaneous family engagement is changing the perception and reality of virtual healthcare. These services need the collaboration of stakeholders across healthcare.

The use of VR for physicians should include certificated training to improve skills and competences in the use of new technology. The "medical virtualist" specialist will cover the aspects of nonphysical examination therapies. Furthermore, new therapies derived from quarantine isolation will consider new approaches for the relationship between physicians and patients.

\section{SUMMARY AND CONCLUSIONS}

The COVID-19 pandemic represents the worst global natural disaster of our generation. As this article suggests, the COVID-19 pandemic is negatively impacting the general population, especially frontline workers, isolated older adults, and those with mental health issues. It is leading to mental health issues in the general public and is exacerbating problems for those already suffering from mental health issues. In this article, we offer the most up-to-date review of the effects of COVID-19 confinement on all the disorders listed in the DSM and make data-driven predictions of the impact of COVID-19 confinement on each. We discuss the potential of digital therapeutic technologies, such as video-based telehealth and immersive VR that offer cost-effective, scalable, on-demand solution to address these mental health problems as part of a multipronged approach. The time to act is now. We need to act decisively and collaboratively. We are all vulnerable to suffer from mental illness and health problems. We can all benefit from establishing and improving access to new ways to tackle these problems, and digital therapeutic technologies are available to help.

\section{Financial support and sponsorship}

RSF and JMGM were employed by Psious, at the time of submission. KV was funded by the European Commission i) within the funding framework of NEURON under the ERA-NET scheme (consortium THERENIA, with funds of BMBF, Germany, 01GP1822) and ii) within the funding framework of Horizon 2020 under the FET Proactive scheme (consortium VIRTUALTIMES, grant agreement 824128). TF and TM are employed by IKONA.

\section{Conflicts of interest}

Psious and IKONA are privately owned digital health companies.

\section{REFERENCES}

1. InformationforHealthcareProfessionalsaboutCoronavirus(COVID-19) CDC. Available from: https://www.cdc.gov/coronavirus/2019-nCoV/ hcp/index.html. [Last accessed on 2020 May 18].

2. Coronavirus (COVID-19) Events as They Happen. Available from: https:/www.who.int/emergencies/diseases/novel-coronavirus-2019/ events-as-they-happen. [Last accessed on 2020 May 18].

3. WHO COVID-19 Strategy Update; 2020.

4. Hawryluck L, Gold WL, Robinson S, Pogorski S, Galea S, Styra R. SARS control and psychological effects of quarantine, Toronto, Canada. Emerg Infect Dis 2004;10:1206-12.

5. Sprang G, Silman M. Posttraumatic stress disorder in parents and youth after health-related disasters. Disaster Med Public Health Prep 2013;7:105-10.

6. BeagleholeB, Mulder RT, Frampton CM, BodenJM,Newton-Howes G, Bell CJ. Psychological distress and psychiatric disorder after natural disasters: Systematic review and meta-analysis. Br J Psychiatry 2018;213:716-22.

7. Brooks SK, Webster RK, Smith LE, Woodland L, Wessely S, Greenberg N, et al. The psychological impact of quarantine and how to reduce it: Rapid review of the evidence. Lancet 2020;395:912-20.

8. Hossain MM, Sultana A, Purohit N. Mental health outcomes of quarantine and isolation for infection prevention: A systematic umbrella review of the global evidence. Epidemiol Health 2020;42:e2020038.

9. Holmes EA, O'Connor RC, Perry VH, Tracey I, Wessely S, Arseneault L, et al. Multidisciplinary research priorities for the COVID-19 pandemic: A call for action for mental health science. Lancet Psychiatry 2020;7:547-60.

10. Huang $\mathrm{Y}$, Zhao N. Generalized anxiety disorder, depressive symptoms and sleep quality during COVID-19 outbreak in China: A web-based cross-sectional survey. Psychiatry Res 2020;288:112954.

11. Rosen Z, Weinberger-Litman SL, Rosenzweig C, Rosmarin DH, Muennig P, Carmody ER, et al. Anxiety and distress among the first community quarantined in the U.S due to COVID-19: Psychological implications for the unfolding crisis. J Chem Inf Model 2020;53:1689-99.

12. Liu S, Yang L, Zhang C, Xiang YT, Liu Z, Hu S, et al. Online mental health services in China during the COVID-19 outbreak. Lancet Psychiatry 2020;7:e17-8.

13. Galea S, Merchant RM, Lurie N. The mental health consequences of COVID-19 and physical distancing: The need for prevention and early intervention. JAMA Intern Med 2020;180:817-8.

14. Odriozola-González P, Planchuelo-Gómez Á, Irurtia-Muñiz MJ, 


\section{Sainz-Fuertes, et al.: COVID-19 and mental health}

Luis-García de R. Psychological symptoms of the outbreak of the COVID-19 crisis and confinement in the population of Spain. Journal of Health Psychology 2020:1359105320967086.

15. Rogers JP, Chesney E, Oliver D, Pollak TA, McGuire P, Fusar-Poli P, et al. Psychiatric and neuropsychiatric presentations associated with severe coronavirus infections: A systematic review and meta-analysis with comparison to the COVID-19 pandemic. Lancet Psychiatry 2020;7:611-27.

16. Banerjee D. The COVID-19 outbreak: Crucial role the psychiatrists can play. Asian J Psychiatr 2020;50:102014.

17. The Royal College of Psychiatrists. PIPSIG Guidelines for the use of Telepsychiatry; 2015. Available from: https://www.rcpsych. ac.uk/docs/default-source/members/sigs/private-and-independentpractice-pipsig/pipsig-telepsychiatry-guidelines-revised-mar16.pdf. [Last accessed on 2020 May 18].

18. Telepsychiatry. Available from: https://www.psychiatry.org/ psychiatrists/practice/telepsychiatry. [Last accessed on 2020 May 18].

19. WHO/Europe Mental Health and COVID-19. Available from: https:// www.euro.who.int/en/health-topics/noncommunicable-diseases/ mental-health/data-and-resources/mental-health-and-covid-19. [Last accessed on 2020 Jun 16].

20. Centers for Disease Control and Prevention. Mental Health and Coping During COVID-19 CDC. Centers for Disease Control and Prevention. Available from: https://www.cdc.gov/coronavirus/2019ncov/daily-life-coping/managing-stress-anxiety.html. Published 2020. [Last accessed on 2020 Jun 16].

21. National Autistic Society. Coronavirus (COVID-19) advice National Autistic Society. Available from: https://www.autism.org.uk/ services/nas-schools/vanguard/news/2020/march/coronavirus(covid-19)-advice.aspx. [Last accessed on 2020 Jun 17].

22. Cortese S, Asherson P, Sonuga-Barke E, Banaschewski T, Brandeis D, Buitelaar J, et al. ADHD management during the COVID-19 pandemic: Guidance from the European ADHD Guidelines Group. Lancet Child Adolesc Health 2020;4:412-4.

23. Foundation CB. COVID-19 - Information and Resources. Available from: https://www.challengingbehaviour.org.uk/information/ covid19information.html. [Last accessed on 2020 Jun 17].

24. Lee J. Mental health effects of school closures during COVID-19. Lancet Child Adolesc Health 2020;4:421.

25. Fonseca L, Diniz E, Mendonça G, Malinowski F, Mari J, Gadelha A. Schizophrenia and COVID-19: Risks and recommendations. Braz J Psychiatry 2020;42:236-8.

26. Brown E, Gray R, Lo Monaco S, O'Donoghue B, Nelson B, Thompson A, et al. The potential impact of COVID-19 on psychosis: A rapid review of contemporary epidemic and pandemic research. Schizophr Res 2020;222:79-87.

27. Eric Youngstrom PD, Hinshaw SP, Stefana A, Chen J, Michael K, Meter AV, et al. Working with bipolar disorder during the COVID-19 pandemic: Both crisis and opportunity. WikiJournal of Medicine 2020;7:4.

28. Javelot H, Llorca PM, Drapier D, Fakra E, Hingray C, Meyer G, et al. Informations on psychotropics and their adaptations for patients suffering from mental disorders in France during the SARS-CoV-2 epidemic. Encephale 2020;46:S14-34.

29. Stefana A, Youngstrom EA, Jun C, Hinshaw S, Maxwell V, Michalak $\mathrm{E}$, et al. The COVID-19 pandemic is a crisis and opportunity for bipolar disorder. Bipolar Disord 2020;22:641-3.

30. Managing my Mental Health During COVID-19 International Bipolar Foundation. Available from: https://ibpf.org/managing-mymental-health-during-covid-19/. [Last accessed on 2020 Jun 16].

31. Key Links to Help you During the Covid-19 Pandemic Bipolar UK. Available from: https://www.bipolaruk.org/blog/key-links-tohelp-you-during-the-covid-19-pandemic. [Last accessed on 2020 Jun 16].
32. Too LS, Spittal MJ, Bugeja L, Reifels L, Butterworth P, Pirkis J. The association between mental disorders and suicide: A systematic review and meta-analysis of record linkage studies. J Affect Disord 2019;259:302-13.

33. Weems CF, Mccurdy B, Carrion V, Scozzafava MD. Increased risk of suicide due to economic and social impacts of social distancing measures to address the Covid-19 pandemic: A forecast. Res Prepr 2020. doi:10.13140/RG.2.2.21601.45926.

34. Yip PS, Cheung YT, Chau PH, Law YW. The impact of epidemic outbreak: The case of severe acute respiratory syndrome (SARS) and suicide among older adults in Hong Kong. Crisis 2010;31:86-92.

35. Fineberg NA, Van Ameringen M, Drummond L, Hollander E, Stein DJ, Geller D, et al. How to manage obsessive-compulsive disorder (OCD) under COVID-19: A clinician's guide from the international college of obsessive compulsive spectrum disorders (ICOCS) and the obsessive-compulsive research network (OCRN) of the European college of neuropsychopharmacology. Compr Psychiatry 2020;100:152174.

36. Horesh D, Brown AD. Covid-19 response: Traumatic stress in the age of Covid-19: A call to close critical gaps and adapt to new realities. Psychol Trauma Theory Res Pract Policy 2020;12:331-5.

37. Qiu J, Shen B, Zhao M, Wang Z, Xie B, Xu Y. A nationwide survey of psychological distress among Chinese people in the COVID-19 epidemic: Implications and policy recommendations. Gen Psychiatry 2020;33:61-3.

38. Zhang J, Lu H, Zeng H, Zhang S, Du Q, Jiang T, et al. The differential psychological distress of populations affected by the COVID-19 pandemic. Brain Behav Immun 2020;87:49-50.

39. Touyz S, Lacey H, Hay P. Eating disorders in the time of COVID-19. J Eat Disord 2020;8:19.

40. Barakat S, Maguire S, Surgenor L, Donnelly B, Miceska B, Fromholtz $\mathrm{K}$, et al. The role of regular eating and self-monitoring in the treatment of bulimia nervosa: a pilot study of an online guided self-help CBT program. Behav Sci (Basel) 2017;7:39.

41. Ohayon MM. Epidemiology of insomnia: What we know and what we still need to learn. Sleep Med Rev 2002;6:97-111.

42. Xie X, Xue Q, Zhou Y, Zhu K, Song R. Mental health status among children in home confinement during the coronavirus disease 2019 outbreak in hubei province, China. JAMA Pediatr 2020;174:898-900.

43. Bhat S, Chokroverty S. Sleep disorders and COVID-19. Sleep medicine 2021. DOI: 10.1016/j.sleep.2021.07.021.

44. COVID-19: Potential Implications for Individuals with Substance Use Disorders Nora's Blog, NIDA. Available from: https:/www. drugabuse.gov/about-nida/noras-blog/2020/04/covid-19-potentialimplications-individuals-substance-use-disorders. [Last accessed on 2020 May 19].

45. Panchal N, Kamal R, Orgera K, Cox C, Garfield R. The Implications of COVID-19 for Mental Health and Substance Use; 2020. Available from: https://www.kff.org/coronavirus-covid-19/issue-brief/theimplications-of-covid-19-for-mental-health-and-substance-use/. [Last accessed on 2020 May 20].

46. Brown EE, Kumar S, Rajji TK, Pollock BG, Mulsant BH. Anticipating and mitigating the impact of the COVID-19 pandemic on Alzheimer's Disease and related dementias. Am J Geriatr Psychiatry 2020;28:712-21.

47. Wang $\mathrm{H}, \mathrm{Li}$, Barbarino $\mathrm{P}$, Gauthier S, Brodaty $\mathrm{H}$, Molinuevo JL, et al. Dementia care during COVID-19. Lancet 2020;395:1190-1.

48. Gunnell D, Appleby L, Arensman E, Hawton K, John A, Kapur N, et al. Suicide risk and prevention during the COVID-19 pandemic. Lancet Psychiatry 2020;7:468-71.

49. Nobles J, Martin F, Dawson S, Moran P, Savovic J. The potential impact of COVID-19 on mental health outcomes and the implications for service solutions. Natl Inst Heal Res 2020. Available from: https:/ /arc-w.nihr.ac.uk/research-and-implementation/covid-19-response/ 


\section{Sainz-Fuertes, et al.: COVID-19 and mental health}

reports/potential-impact-of-covid-19-on-mental-health-outcomesand-the-implications-for-service-solutions/. [Lass accessed on 2020 Aug 30].

50. Zhou X, Snoswell CL, Harding LE, Bambling M, Edirippulige S, Bai X, et al. The role of telehealth in reducing the mental health burden from COVID-19. Telemed e-Health 2020;26:377-9.

51. Maddox T, Fitzpatrick T. The promise of virtual reality in health-care education and training: It's all in the neuroscience. Digital Health 2020;5:133-7.

52. Pizzoli SFM, Mazzocco K, Triberti S, Monzani D, Alcañiz Raya ML, Pravettoni G. User-centered virtual reality for promoting relaxation: An innovative approach. Front Psychol 2019;10:479.

53. Rizzo A', Shilling R. Clinical virtual reality tools to advance the prevention, assessment, and treatment of PTSD. Eur J Psychotraumatol 2017;8:1414560.

54. Oing T, Prescott J. Implementations of virtual reality for anxietyrelated disorders: Systematic review. JMIR Serious Games 2018;6:e10965.
55. Darnall BD, Krishnamurthy P, Tsuei J, Minor JD. Self-administered skills-based virtual reality intervention for chronic Pain: Randomized controlled pilot study. JMIR Form Res 2020;4:e17293.

56. Ghiță A, Hernández-Serrano O, Fernández-Ruiz $\mathrm{Y}$, Monras M, Ortega L, Mondon S, et al. Cue-elicited anxiety and alcohol craving as indicators of the validity of ALCO-VR software: A virtual reality study. J Clin Med 2019;8:1153.

57. Freeman D, Yu LM, Kabir T, Martin J, Craven M, Leal J, et al. Automated virtual reality (VR) cognitive therapy for patients with psychosis: Study protocol for a single-blind parallel group randomised controlled trial (gameChange). BMJ Open 2019;9:e031606.

58. Villani D, Rotasperti C, Cipresso P, Triberti S, Carissoli C, Riva G. Assessing the emotional state of job applicants through a virtual reality simulation: A psycho-physiological study. eHealth $360^{\circ}$. Springer, Cham, 2017. p. 119-26.

59. Skinner BF. The Behavior of Organisms: An Experimental Analysis. New York: Appleton-Century; 1938.

60. Nochomovitz M, Sharma R. Is it time for a new medical specialty? The medical virtualist. JAMA 2018;319:437-8. 